\title{
ON THE SHAPE FIELDS FINITENESS PRINCIPLE
}

\author{
FUSHUAI JIANG, GARVING K. LULI, AND KEVIN O'NEILL
}

\begin{abstract}
In this paper, we improve the finiteness constant for the finiteness principles for $\mathrm{C}^{\mathrm{m}}\left(\mathbb{R}^{\mathrm{n}}, \mathbb{R}^{\mathrm{d}}\right)$ and $\mathrm{C}^{\mathrm{m}-1,1}\left(\mathbb{R}^{\mathrm{n}}, \mathbb{R}^{\mathrm{D}}\right)$ selection proven in 19] and extend the more general shape fields finiteness principle to the vector-valued case.
\end{abstract}

\section{INTRODUCTION}

Suppose we are given integers $m \geq 0, n \geq 1, D \geq 1$. We write $C^{m}\left(\mathbb{R}^{n}, \mathbb{R}^{D}\right)$ to denote the space of all functions $\overrightarrow{\mathrm{F}}: \mathbb{R}^{n} \rightarrow \mathbb{R}^{\mathrm{D}}$ whose derivatives $\partial^{\alpha} \overrightarrow{\mathrm{F}}$ (for all $|\alpha| \leq \mathrm{m})$ are continuous and bounded on $\mathbb{R}^{n}$, equipped with the norm

$$
\|\vec{F}\|_{C^{m}\left(\mathbb{R}^{n}, \mathbb{R}^{D}\right)}:=\max _{|\alpha| \leq m} \sup _{x \in \mathbb{R}^{n}}\left\|\partial^{\alpha} \vec{F}(x)\right\|_{\infty}=\max _{\substack{|\alpha| \leq m \\ 1 \leq j \leq D}} \sup _{x \in \mathbb{R}^{n}}\left|\partial^{\alpha} F_{j}(x)\right| .
$$

Here and below, we view $\partial^{\alpha} \vec{F}(x)=\left(\partial^{\alpha} F_{1}(x), \cdots, \partial^{\alpha} F_{D}(x)\right)$ as a vector in $\mathbb{R}^{D}$.

We write $\dot{C}^{m}\left(\mathbb{R}^{n}, \mathbb{R}^{D}\right)$ to denote the vector space of $m$-times continuously differentiable $\mathbb{R}^{\mathrm{D}}$-valued functions whose $\mathrm{m}$-th order derivatives are bounded, equipped with the seminorm

$$
\|\overrightarrow{\mathrm{F}}\|_{\dot{\mathrm{C}}^{\mathrm{m}}\left(\mathbb{R}^{n}, \mathbb{R}^{\mathrm{D}}\right)}:=\max _{|\alpha|=\mathrm{m}} \sup _{x \in \mathbb{R}^{n}}\left\|\partial^{\alpha} \overrightarrow{\mathrm{F}}(x)\right\|_{\infty} .
$$

We write $C^{m-1,1}\left(\mathbb{R}^{n}, \mathbb{R}^{\mathrm{D}}\right)$ to denote the space of all $\overrightarrow{\mathrm{F}}: \mathbb{R}^{n} \rightarrow \mathbb{R}^{\mathrm{D}}$ whose derivatives $\partial^{\alpha} \vec{F}$ (for all $|\alpha| \leq m-1$ ) are bounded and Lipschitz on $\mathbb{R}^{n}$. When $D=1$, we write $C^{m}\left(\mathbb{R}^{n}\right)$ and $C^{m-1,1}\left(\mathbb{R}^{n}\right)$ in place of $C^{m}\left(\mathbb{R}^{n}, \mathbb{R}^{D}\right)$ and $C^{m-1,1}\left(\mathbb{R}^{n}, \mathbb{R}^{D}\right)$.

We write $\overrightarrow{\mathcal{P}}$ to denote the vector space $\bigoplus_{j=1}^{\mathrm{D}} \mathcal{P}$, where $\mathcal{P}$ is the space of polynomials on $\mathbb{R}^{n}$ with degree no greater than $m-1$. Note that $\operatorname{dim} \overrightarrow{\mathcal{P}}=\mathrm{D} \cdot\left(\begin{array}{c}n+m-1 \\ m-1\end{array}\right)$.

Quantities $c(m, n), C(m, n), k(m, n)$, etc., denote constants depending only on $m, n$; these expressions may denote different constants in different occurrences. Similar conventions apply to constants denoted by $C(m, n, D), k(m, n, D)$, etc.

If $S$ is any finite set, then $|S|$ denotes the number of elements in $S$.

Let $E \subset \mathbb{R}^{n}$ be given. Suppose at each $x \in E$, we are given a convex set $K(x) \in \mathbb{R}^{D}$. A selection of $(K(x))_{x \in E}$ is a map $\vec{F}: \mathbb{R}^{n} \rightarrow \mathbb{R}^{D}$ such that $\vec{F}(x) \in K(x)$ for all $x \in \mathrm{E}$.

We are interested in the following selection problem.

Problem 1.1. Let $\mathrm{E} \subset \mathbb{R}^{n}$. For each $\mathrm{x} \in \mathrm{E}$, suppose we are given a convex $\mathrm{K}(\mathrm{x}) \subset \mathbb{R}^{\mathrm{n}}$. Given a number $\mathrm{M}>0$, how can decide if there exists a selection $\overrightarrow{\mathrm{F}} \in \mathrm{C}^{\mathrm{m}-1,1}\left(\mathbb{R}^{\mathrm{n}}, \mathbb{R}^{\mathrm{D}}\right)$ or $\overrightarrow{\mathrm{F}} \in \mathrm{C}^{\mathrm{m}}\left(\mathbb{R}^{\mathrm{n}}, \mathbb{R}^{\mathrm{D}}\right)$ with $\|\overrightarrow{\mathrm{F}}\|_{\mathrm{C}^{\mathrm{m}}\left(\mathbb{R}^{n}, \mathbb{R}^{\mathrm{D}}\right)} \leq \mathrm{C}^{\#} \mathrm{M}$ or $\|\overrightarrow{\mathrm{F}}\|_{\mathrm{C}^{\mathrm{m}-1,1\left(\mathbb{R}^{n}, \mathbb{R}^{\mathrm{D}}\right)}} \leq \mathrm{C}^{\#} \mathrm{M}$, where $\mathrm{C}^{\#}$ depends only on $\mathrm{m}, \mathrm{n}, \mathrm{D}$ ? 
In [19, the authors addressed Problem 1.1 by proving the following

Theorem 1.2 (Finiteness Principle for Smooth Selection). For large enough $\mathrm{k}^{\sharp}=$ $\mathrm{k}(\mathrm{m}, \mathrm{n}, \mathrm{D})$ and $\mathrm{C}^{\#}=\mathrm{C}(\mathrm{m}, \mathrm{n}, \mathrm{D})$, the following hold.

(A) $\mathrm{C}^{\mathrm{m}}$ FLAVOR: Let $\mathrm{E} \subset \mathbb{R}^{\mathrm{n}}$ be finite. For each $\mathrm{x} \in \mathrm{E}$, let $\mathrm{K}(\mathrm{x}) \subset \mathbb{R}^{\mathrm{D}}$ be convex. Suppose that for each $\mathrm{S} \subset \mathrm{E}$ with $|\mathrm{S}| \leq \mathrm{k}^{\sharp}$, there exists $\overrightarrow{\mathrm{F}}^{\mathrm{S}} \in$ $\mathrm{C}^{\mathrm{m}}\left(\mathbb{R}^{\mathrm{n}}, \mathbb{R}^{\mathrm{D}}\right)$ with norm $\left\|\overrightarrow{\mathrm{F}}^{\mathrm{S}}\right\|_{\mathrm{C}^{\mathrm{m}}\left(\mathbb{R}^{n}, \mathbb{R}^{\mathrm{D}}\right)} \leq 1$, such that $\overrightarrow{\mathrm{F}}^{\mathrm{S}}(\mathrm{x}) \in \mathrm{K}(\mathrm{x})$ for all $x \in \mathrm{S}$.

Then there exists $\overrightarrow{\mathrm{F}} \in \mathrm{C}^{\mathrm{m}}\left(\mathbb{R}^{\mathrm{n}}, \mathbb{R}^{\mathrm{D}}\right)$ with norm $\|\overrightarrow{\mathrm{F}}\|_{\mathrm{C}^{\mathrm{m}\left(\mathbb{R}^{n}, \mathbb{R}^{\mathrm{D}}\right)}} \leq \mathrm{C}^{\#}$, such that $\overrightarrow{\mathrm{F}}(\mathrm{x}) \in \mathrm{K}(\mathrm{x})$ for all $\mathrm{x} \in \mathrm{E}$.

(B) $\mathrm{C}^{\mathrm{m}-1,1}$ FLAVOR: Let $\mathrm{E} \subset \mathbb{R}^{\mathrm{n}}$ be arbitrary. For each $\mathrm{x} \in \mathbb{R}^{\mathrm{n}}$, let $\mathrm{K}(\mathrm{x}) \subset \mathbb{R}^{\mathrm{D}}$ be a closed convex set. Suppose that for each $\mathrm{S} \subset \mathrm{E}$ with $|\mathrm{S}| \leq \mathrm{k}^{\sharp}$, there exists $\overrightarrow{\mathrm{F}}^{\mathrm{S}} \in \mathrm{C}^{\mathrm{m}-1,1}\left(\mathbb{R}^{\mathrm{n}}, \mathbb{R}^{\mathrm{D}}\right)$ with norm $\left\|\overrightarrow{\mathrm{F}}^{\mathrm{S}}\right\|_{\mathrm{C}^{\mathrm{m}-1,1}\left(\mathbb{R}^{n}, \mathbb{R}^{\mathrm{D}}\right)} \leq 1$, such that $\overrightarrow{\mathrm{F}}^{\mathrm{S}}(\mathrm{x}) \in \mathrm{K}(\mathrm{x})$ for all $\mathrm{x} \in \mathrm{S}$.

Then there exists $\overrightarrow{\mathrm{F}} \in \mathrm{C}^{\mathrm{m}-1,1}\left(\mathbb{R}^{\mathrm{n}}, \mathbb{R}^{\mathrm{D}}\right)$ with norm $\|\overrightarrow{\mathrm{F}}\|_{\mathrm{C}^{m-1,1}\left(\mathbb{R}^{n}, \mathbb{R}^{\mathrm{D}}\right)} \leq$ $\mathrm{C}^{\#}$, such that $\overrightarrow{\mathrm{F}}(\mathrm{x}) \in \mathrm{K}(\mathrm{x})$ for all $\mathrm{x} \in \mathbb{R}^{\mathrm{n}}$.

Therefore, Theorem 1.2 tells us when there exists a $\mathrm{C}^{\mathrm{m}-1,1}$ selection $\overrightarrow{\mathrm{F}}$ of $(\mathrm{K}(\mathrm{x}))_{\mathrm{x} \in \mathrm{E}}$ for the case of infinite $E$ and provides estimates for the $\mathrm{C}^{\mathrm{m}}$-norm of a selection for finite $\mathrm{E}$.

Theorem 1.2 for the case $\mathrm{D}=1$ and $\mathrm{K}(\mathrm{x})$ being a singleton for each $\mathrm{x} \in \mathrm{E}$ was conjectured by Y. Brudnyi and P. Shvartsman in [6].

The number $k^{\sharp}$ in Theorem 1.2 is called the finiteness number. The $k^{\sharp}$ obtained in [19] is $k^{\sharp}=100+(D+2)^{l_{*}+100}$, where $l_{*}=\left(\begin{array}{c}m+n \\ n\end{array}\right)$.

Here, we give a sharper bound on $k^{\sharp}$. Our first result is the following.

Theorem 1.3. The $\mathrm{k}^{\sharp}$ found in Theorem 1.2 may be taken to be $\mathrm{k}^{\sharp}=2^{\operatorname{dim} \overrightarrow{\mathcal{P}}}$, where $\operatorname{dim} \overrightarrow{\mathcal{P}}=\mathrm{D} \cdot\left(\begin{array}{c}\mathrm{n}+\mathrm{m}-1 \\ \mathrm{~m}-1\end{array}\right)$.

A few remarks on Theorem 1.3 are in order. For $\mathrm{D}=1$, our result coincides with the one proven by P. Shvartsman 27. A similar result was obtained by E. Bierstone and P. Milman [1] in the case where each $\mathrm{K}(\mathrm{x})$ consists of a single point, corresponding to the finiteness principle proven by C. Fefferman in [12. Our present approach is inspired by [1].

In the case $\mathrm{D}=1$ and $\mathrm{m}=2$, Theorem 1.3 gives $\mathrm{k}^{\sharp}=4 \cdot 2^{\mathrm{n}-1}$. This is comparable to the finiteness constant $3 \cdot 2^{\text {n-1 }}$ given by Shvartsman [25], which he shows to be optimal. See also 9 .

To prove Theorem [1.3 we will need to extend the finiteness principle proven in [19] to the vector valued case.

Theorem 1.4. The following holds for $\mathbb{X}=\mathrm{C}^{\mathrm{m}}\left(\mathbb{R}^{\mathrm{n}}, \mathbb{R}^{\mathrm{D}}\right)$ and $\mathbb{X}=\dot{\mathrm{C}}^{\mathrm{m}}\left(\mathbb{R}^{\mathrm{n}}, \mathbb{R}^{\mathrm{D}}\right)$.

Let $\mathbf{S} \subset \mathbb{R}^{n}$ be a finite set of diameter at most 1 . For each $\mathrm{x} \in \mathrm{S}$, let $\overrightarrow{\mathrm{G}}(\mathrm{x}) \subset \overrightarrow{\mathcal{P}}$ be convex. Suppose that for every subset $\mathrm{S}^{\prime} \subset \mathrm{S}$ with $|\mathrm{S}| \leq 2^{\operatorname{dim} \overrightarrow{\mathcal{P}}}$, there exists $\mathrm{F}^{\mathrm{S}^{\prime}} \in \mathbb{X}$ such that $\left\|\mathrm{F}^{\mathrm{S}^{\prime}}\right\|_{\mathbb{X}} \leq 1$ and $\mathcal{J}_{\mathrm{x}} \mathrm{F}^{\mathrm{S}^{\prime}} \in \overrightarrow{\mathrm{G}}(\mathrm{x})$ for all $\mathrm{x} \in \mathrm{S}^{\prime}$.

Then, there exists $\mathrm{F} \in \mathbb{X}$ such that $\|\mathrm{F}\|_{\mathbb{X}} \leq \gamma$ and $\mathcal{J}_{x} \mathrm{~F} \in \overrightarrow{\mathrm{G}}(\mathrm{x})$ for all $\mathrm{x} \in \mathrm{S}$.

Here, $\gamma$ depends only on $\mathrm{m}, \mathrm{n}, \mathrm{D}$, and $|\mathrm{S}|$. 
Because the constant $\gamma$ depends on the number of points in S, following [27, we will refer to Theorem 1.4 as a "weak finiteness principle".

To conclude the introduction, we give an overview of how we prove Theorems 1.3 and 1.4. The proof of Theorem 1.2 given in [19 is via a more general finiteness principle for shape fields, see Theorem 2.4 below. Using Theorem 1.4, we will show an improved bound for $k^{\sharp}$ in the finiteness principle for shape fields (i.e., Theorem 2.4); we can then deduce the bound for $k^{\sharp}$ in Theorem 1.2, obtaining the bound asserted in Theorem 1.3. The heart of the matter therefore lies in Theorem 1.4. To put things in perspective, we would like to point out that one can't directly apply the techniques from [1] because of the nonlinear structure in the selection problem and that the result in 27] is for scalar-valued functions. To prove our main theorem (Theorem 1.4), we will adapt the strategy from [1] with some new ingredients: Instead of linear structure, we will handle general convex structure using the duality theorem of linear programming to describe the relevant convex sets.

This paper is part of a literature on extension, interpolation, and selection of functions, going back to H. Whitney's seminal work [29]31, and including fundamental contributions by G. Glaeser 22, Y. Brudnyi and P. Shvartsman 4 9, 25, 26, 28, and E. Bierstone, P. Milman, and W. Pawłucki 1-3], and C. Fefferman [10, 18,21 .

Acknowledgement. We are indebted to Pavel Shvartsman for his valuable comments.

The first author is supported by the UC Davis Summer Graduate Student Researcher Award and the Alice Leung Scholarship in Mathematics. The second author is supported by NSF Grant DMS-1554733 and the UC Davis Chancellor's Fellowship

\section{BACKGROUND AND MAIN RESULTS}

2.1. Polynomial and Whitney fields. We write $\mathcal{P}$ to denote the vector space of polynomials on $\mathbb{R}^{n}$ with degree no greater than $m-1$.

For $x \in \mathbb{R}^{n}$, let $F$ be $(m-1)$-times differentiable at $x$. We identify the $(m-1)$-jet of $F$ at $x$ with the $(m-1)^{\text {st }}$-degree Taylor polynomial of $F$ at $x$ :

$$
\partial_{x} F(y):=\sum_{|\alpha| \leq m-1} \frac{\partial^{\alpha} F(x)}{\alpha !}(y-x)^{\alpha} .
$$

For $\mathrm{P}, \mathrm{Q} \in \mathcal{P}$ and $x \in \mathbb{R}^{n}$, we define

$$
\mathrm{P} \odot_{x} \mathrm{Q}:=\mathcal{J}_{x}(\mathrm{PQ}) .
$$

The operation $\odot_{x}$ turns $\mathcal{P}$ into a ring, which we denote by $\mathcal{R}_{\chi}$.

We define

$$
\overrightarrow{\mathcal{P}}:=\underbrace{\mathcal{P} \oplus \cdots \oplus \mathcal{P}}_{\text {D copies }} .
$$

Thus, every $\vec{P} \in \overrightarrow{\mathcal{P}}$ has the form $\vec{P}=\left(P_{1}, \cdots, P_{D}\right)$, with $P_{j} \in \mathcal{P}$ for $j=1, \cdots, D$.

Let $\vec{F}=\left(F_{1}, \cdots, F_{D}\right)$ be a $\mathbb{R}^{D}$-valued function $(m-1)$-times differentiable at $x \in \mathbb{R}^{n}$. We define

$$
\mathcal{J}_{x} \vec{F}:=\left(\mathcal{J}_{x} F_{1}, \cdots, \mathcal{J}_{x} F_{D}\right) \in \overrightarrow{\mathcal{P}}
$$


We will also use the $\mathcal{R}_{\chi}$-module structure on $\overrightarrow{\mathcal{P}}$, whose multiplication is given by

$$
\mathrm{R} \odot_{x} \overrightarrow{\mathrm{P}}:=\left(\mathrm{R} \odot_{x} \mathrm{P}_{1}, \cdots, \mathrm{R} \odot_{x} \mathrm{P}_{\mathrm{D}}\right) \in \overrightarrow{\mathcal{P}},
$$

for $x \in \mathbb{R}^{n}, \vec{P}=\left(P_{1}, \cdots, P_{D}\right) \in \overrightarrow{\mathcal{P}}$, and $R \in \mathcal{R}_{x}$.

Let $S \subset \mathbb{R}^{n}$ be a finite set. A Whitney field is an array $\left(\overrightarrow{\mathrm{P}}^{\mathrm{x}}\right)_{x \in S}$ parameterized by points in $S$, where $\overrightarrow{\mathrm{P}}^{x} \in \overrightarrow{\mathcal{P}}$ for $x \in S$. We write $W^{m}(S)$ to denote the space of Whitney fields on $S$.

Given $\left(\overrightarrow{\mathrm{P}}^{\mathrm{x}}\right)_{\mathrm{x} \in \mathrm{S}} \in \mathrm{W}^{\mathrm{m}}(\mathrm{S})$, we define

$$
\left\|\left(\vec{P}^{x}\right)_{x \in S}\right\|_{W^{m}(S)}:=\max _{\substack{x \in S \\|\alpha| \leq m-1}}\left\|\partial^{\alpha} \vec{P}^{x}(x)\right\|_{\infty}+\max _{\substack{x, y \in S, x \neq y \\|\alpha| \leq m-1}} \frac{\left\|\partial^{\alpha}\left(\vec{P}^{x}-\vec{P}^{y}\right)(x)\right\|_{\infty}}{|x-y|^{m-|\alpha|}} .
$$

Note that $\|\cdot\|_{W^{m}(S)}$ is a norm on $W^{m}(S)$.

We will also be using the seminorm

$$
\left\|\left(\overrightarrow{\mathrm{P}}^{x}\right)_{x \in S}\right\|_{\dot{W}^{m}(S)}:=\max _{\substack{x, y \in S, x \neq y \\|\alpha| \leq m-1}} \frac{\left\|\partial^{\alpha}\left(\overrightarrow{\mathrm{P}}^{x}-\overrightarrow{\mathrm{P}}^{y}\right)(x)\right\|_{\infty}}{|x-y|^{m-|\alpha|}} .
$$

We use $\overrightarrow{\mathcal{P}}^{*}$ to denote the dual of $\overrightarrow{\mathcal{P}}$. We use $W^{m}(S)^{*}$ to denote the dual of $W^{m}(S)$. An element $\xi \in W^{m}(S)^{*}$ has the form $\xi=\left(\xi_{x}\right)_{x \in S}$. We use $\xi\left[\left(\vec{P}^{x}\right)_{x \in S}\right]$ to denote the action of $\xi \in W^{\mathrm{m}}(S)^{*}$ on $\left(\overrightarrow{\mathrm{P}}^{\mathrm{x}}\right)_{\mathrm{x} \in \mathrm{S}} \in \mathrm{W}^{\mathrm{m}}(\mathrm{S})$.

Lemma 2.1. Fix $\mathrm{m}, \mathrm{n}, \mathrm{D} \in \mathbb{N}$. There exists $\mathrm{C}=\mathrm{C}(\mathrm{m}, \mathrm{n})<\infty$ such that the following hold.

(1) Let $\mathrm{S} \subset \mathbb{R}^{n}$ be a finite set.

(a) For all $\overrightarrow{\mathrm{F}} \in \mathrm{C}^{\mathrm{m}}\left(\mathbb{R}^{\mathrm{n}}, \mathbb{R}^{\mathrm{D}}\right),\left\|\left(\mathcal{J}_{x} \overrightarrow{\mathrm{F}}\right)_{x \in S}\right\|_{W^{\mathrm{m}}(\mathrm{S})} \leq \mathrm{C}\|\overrightarrow{\mathrm{F}}\|_{\mathrm{C}^{\mathrm{m}}\left(\mathbb{R}^{\mathrm{n}}, \mathbb{R}^{\mathrm{D}}\right)}$.

(b) Let $C_{\text {loc }}^{m}\left(\mathbb{R}^{n}, \mathbb{R}^{\mathrm{D}}\right)$ denotes the space of $\mathbb{R}^{\mathrm{D}}$-valued functions on $\mathbb{R}^{\mathrm{n}}$ with continuous derivatives up to order $\mathrm{m}$. For all $\overrightarrow{\mathrm{F}} \in \mathrm{C}_{\mathrm{loc}}^{\mathrm{m}}\left(\mathbb{R}^{\mathrm{n}}, \mathbb{R}^{\mathrm{D}}\right)$, $\left\|\left(\mathcal{J}_{x} \overrightarrow{\mathrm{F}}\right)_{x \in S}\right\|_{\dot{W}^{m}(S)} \leq \mathrm{C}\|\overrightarrow{\mathrm{F}}\|_{\dot{\mathrm{C}}^{m}\left(\mathbb{R}^{\mathrm{n}}, \mathbb{R}^{\mathrm{D}}\right)}$.

(2) Let $\mathrm{S} \subset \mathbb{R}^{n}$ be a finite set. There exists a linear map $\mathrm{T}_{w}^{\mathrm{S}}: \mathrm{W}^{\mathrm{m}}(\mathrm{S}) \rightarrow$ $\mathrm{C}^{\mathrm{m}}\left(\mathbb{R}^{\mathrm{n}}, \mathbb{R}^{\mathrm{D}}\right)$ such that

(a) $\left\|\mathrm{T}_{w}^{\mathrm{S}}\left[\left(\overrightarrow{\mathrm{P}}^{\mathrm{x}}\right)_{\mathrm{x} \in \mathrm{S}}\right]\right\|_{\mathrm{C}^{\mathrm{m}}\left(\mathbb{R}^{\mathrm{n}}, \mathbb{R}^{\mathrm{D}}\right)} \leq\left\|\left(\overrightarrow{\mathrm{P}}^{\mathrm{x}}\right)_{\mathrm{x} \in \mathrm{S}}\right\|_{W^{\mathrm{m}}(\mathrm{S})}$,

(b) $\left\|\mathrm{T}_{w}^{\mathrm{S}}\left[\left(\overrightarrow{\mathrm{P}}^{\mathrm{x}}\right)_{x \in S}\right]\right\|_{\dot{\mathrm{C}}^{\mathrm{m}}\left(\mathbb{R}^{n}, \mathbb{R}^{\mathrm{D}}\right)} \leq\left\|\left(\overrightarrow{\mathrm{P}}^{\mathrm{x}}\right)_{\mathrm{x} \in \mathrm{S}}\right\|_{\dot{W}^{\mathrm{m}}(\mathrm{S})}$, and

(c) $\mathcal{J}_{x} \circ \mathrm{T}_{w}^{\mathrm{S}}\left[\left(\overrightarrow{\mathrm{P}}^{\mathrm{x}}\right)_{\mathrm{x} \in \mathrm{S}}\right]=\overrightarrow{\mathrm{P}}^{\mathrm{x}}$ for each $\mathrm{x} \in \mathrm{S}$.

Lemma 2.1(1) is simply Taylor's theorem. Lemma 2.1 (2) is an $\mathbb{R}^{\mathrm{D}}$-valued version of the Whitney Extension Theorem (for finite sets), the proof of which follows the $\mathbb{R}$-valued case by component-wise treatment. The proof for the classical Whitney Extension Theorem can be found in e.g. [23.

2.2. Shape fields. In this section, we generalize a key object introduced in [19].

Definition 2.2. Let $S \subset \mathbb{R}^{n}$ be finite. For each $x \in S, 0<M<\infty$, let $\vec{\Gamma}(\mathrm{x}, \mathrm{M}) \subset \overrightarrow{\mathrm{P}}$ be a (possibly empty) convex set. We say that $(\vec{\Gamma}(\mathrm{x}, \mathrm{M}))_{\mathrm{x} \in \mathrm{S}, \mathrm{M}>0}$ is a vector-valued shape field if for all $\mathrm{x} \in \mathrm{S}$ and $0<\mathrm{M}^{\prime} \leq \mathrm{M}<\infty$, we have

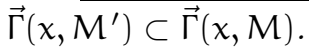

When $\mathrm{D}=1$, we write $\Gamma(\mathrm{x}, \mathrm{M})$ instead of $\vec{\Gamma}(\mathrm{x}, \mathrm{M})$, and we omit the adjective "vector-valued". 
Definition 2.3. Let $\mathrm{C}_{w}, \delta_{\max }$ be positive real numbers. We say that a vector-valued shape field $(\vec{\Gamma}(x, M))_{x \in S, M>0}$ is $\left(C_{w}, \delta_{\max }\right)$-convex if the following condition holds:

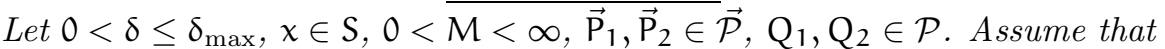

(1) $\vec{P}_{1}, \vec{P}_{2} \in \vec{\Gamma}(x, M)$;

(2) $\left\|\partial^{\alpha}\left(\vec{P}_{1}-\vec{P}_{2}\right)(x)\right\|_{\infty} \leq M \delta^{m-|\alpha|}$ for $|\alpha| \leq m-1$;

(3) $\left|\partial^{\alpha} Q_{i}(x)\right| \leq \delta^{-|\alpha|}$ for $|\alpha| \leq m-1$ and $i=1,2$;

(4) $\mathrm{Q}_{1} \odot_{x} \mathrm{Q}_{1}+\mathrm{Q}_{2} \odot_{x} \mathrm{Q}_{2}=1$. Then

(5) $\overrightarrow{\mathrm{P}}:=\sum_{i=1}^{2}\left(\mathrm{Q}_{i} \odot_{x} \mathrm{Q}_{i}\right) \odot_{x} \overrightarrow{\mathrm{P}}_{\mathrm{i}} \in \vec{\Gamma}\left(x, \mathrm{C}_{w} \mathrm{M}\right)$. Here, in each summand, the first multiplication is the ring multiplication in $\mathcal{R}_{\mathrm{x}}$, and the second is the action of $\mathcal{R}_{x}$ on the $\mathcal{R}_{\mathrm{x}}$-module.

2.3. Main technical results. The main technical results are the following two theorems. The first is the Finiteness Principle for vector-valued shape fields, and second improves the finiteness constant.

Theorem 2.4. There exists $\mathrm{k}^{\sharp}=\mathrm{k}^{\sharp}(\mathrm{m}, \mathrm{n}, \mathrm{D})$ such that the following holds.

Let $\mathrm{E} \subset \mathbb{R}^{\mathrm{n}}$ be a finite set and $(\vec{\Gamma}(\mathrm{x}, \mathrm{M}))_{\mathrm{x} \in \mathrm{E}, \mathrm{M}>0}$ be $a\left(\mathrm{C}_{w}, \delta_{\max }\right)$-convex vectorvalued shape field. Let $\mathrm{Q}_{0} \subset \mathbb{R}^{n}$ be a cube of side length $\delta_{\mathrm{Q}_{0}} \leq \delta_{\max }$ and $\mathrm{x}_{0} \in$ $\mathrm{E} \cap 5 \mathrm{Q}_{0}$ and $\mathrm{M}_{0}>0$ be given.

Suppose that for each $\mathrm{S} \subset \mathrm{E}$ with $|\mathrm{S}| \leq \mathrm{k}^{\sharp}$, there exists a Whitney field $\left(\overrightarrow{\mathrm{P}}^{z}\right)_{z \in \mathrm{S}}$ such that

and

$$
\left\|\left(\overrightarrow{\mathrm{P}}^{z}\right)_{z \in \mathrm{S}}\right\|_{\dot{\mathrm{W}}^{\mathrm{m}}(\mathrm{S})} \leq \mathrm{M}_{0}
$$

Then, there exist $\overrightarrow{\mathrm{P}}^{0} \in \vec{\Gamma}\left(\mathrm{x}_{0}, \mathrm{M}_{0}\right)$ and $\overrightarrow{\mathrm{F}} \in \mathrm{C}^{\mathrm{m}}\left(\mathrm{Q}_{0}, \mathbb{R}^{\mathrm{D}}\right)$ such that

- $\mathrm{J}_{z} \overrightarrow{\mathrm{F}} \in \vec{\Gamma}(z, C M)$ for all $z \in \mathrm{E} \cap 5 \mathrm{Q}_{0}$.

- $\left\|\partial^{\alpha}\left(\overrightarrow{\mathrm{F}}-\overrightarrow{\mathrm{P}}^{0}\right)(\mathrm{x})\right\|_{\infty} \leq \mathrm{CM}_{0} \delta_{\mathrm{Q}_{0}}^{\mathrm{m}-|\alpha|}$ for all $\mathrm{x} \in \mathrm{Q}_{0},|\alpha| \leq \mathrm{m}$.

- In particular, $\left\|\partial^{\alpha} \overrightarrow{\mathrm{F}}(\mathrm{x})\right\|_{\infty} \leq \mathrm{CM}_{0}$ for all $\mathrm{x} \in \mathrm{Q}_{0},|\alpha|=\mathrm{m}$.

The case of scalar-valued shape fields $(\mathrm{D}=1)$ was proven in [19]. In this paper, we will use the $\mathrm{D}=1$ case to prove the more general Theorem 2.4 stated above using a gradient trick, inspired by [19,20].

Theorem 2.5. One may take $\mathrm{k}^{\sharp}=2^{\operatorname{dim} \overrightarrow{\mathcal{P}}}$ in Theorem 2.4.

Proof of Theorem 2.5 via Theorem 1.4. Take as given the hypotheses for Theorem 2.4, but with $k^{\sharp}=2^{\operatorname{dim} \overrightarrow{\mathcal{P}}}$. This means that for each $S^{\prime} \subset E$ with $\left|S^{\prime}\right|=2^{\operatorname{dim} \vec{P}}$, there exists $\left(\overrightarrow{\mathrm{P}}^{z}\right)_{z \in \mathrm{S}^{\prime}}$ such that

$$
\left\|\left(\overrightarrow{\mathrm{P}}^{z}\right)_{z \in S^{\prime}}\right\|_{\dot{W}^{m}\left(S^{\prime}\right)} \leq M_{0}
$$

and

$$
\overrightarrow{\mathrm{P}}^{z} \in \vec{\Gamma}\left(z, \mathrm{M}_{0}\right) .
$$

Recall that in the definition of shape field, we require $\Gamma(x, M)$ be convex for all $x \in S$ and $M>0$.

Let $S \subset E$ with $|S| \leq k^{\sharp}$, where $k^{\sharp}$ is as initially stated in Theorem 2.4 (and coming from [19] and our gradient trick for $\mathrm{D} \geq 2$ ). Then, the above holds for all 
$S^{\prime} \subset S$ with $\left|S^{\prime}\right|=2^{\operatorname{dim} \overrightarrow{\mathcal{P}}}$, so by the homogeneous version of Theorem 1.4, there exists $\overrightarrow{\mathrm{F}} \in \dot{\mathrm{C}}^{\mathrm{m}}\left(\mathbb{R}^{\mathrm{n}}, \mathbb{R}^{\mathrm{D}}\right)$ such that

$$
\|\overrightarrow{\mathrm{F}}\|_{\dot{\mathrm{C}}^{\mathrm{m}}\left(\mathbb{R}^{n}, \mathbb{R}^{\mathrm{D}}\right)} \leq \gamma \mathrm{M}_{0}
$$

and

$$
\mathrm{J}_{x} \overrightarrow{\mathrm{F}} \in \Gamma\left(x, \mathrm{M}_{0}\right) \text { for all } x \in \mathrm{S} \text {. }
$$

By (2.7), we have

$$
\left\|\left(J_{x} \vec{F}^{x}\right)_{x \in S}\right\|_{\dot{W}^{m}(S)} \leq C \gamma M_{0} \text {. }
$$

Thus, the hypotheses for Theorem 2.4 with the $k^{\sharp}$ from the initial statement are satisfied.

At this point, we have shown that the shape fields finiteness principle holds with an improved value of $k^{\sharp}$ (Theorem [2.5); the next step is to show that the selection problem of Theorems 1.3 and 1.4 may be described through shape fields.

Proof of Theorem 1.3 via Theorem 2.5. Let

$$
\vec{\Gamma}(x, M):=\left\{\overrightarrow{\mathrm{P}} \in \overrightarrow{\mathcal{P}}:\left\|\partial^{\alpha} \overrightarrow{\mathrm{P}}(x)\right\|_{\infty} \leq M, \overrightarrow{\mathrm{P}}(x) \in K(x)\right\} .
$$

It suffices to observe that $(\vec{\Gamma}(x, M))_{x \in E, M>0}$ is a $(C, 1)$-convex shape field when $\mathrm{K}(\mathrm{x})$ is convex for each $x \in \mathrm{E}$.

Let $\delta \in(0,1], x \in E, M \in(0, \infty), \vec{P}_{1}, \vec{P}_{2} \in \overrightarrow{\mathcal{P}}$, and $\mathrm{Q}_{1}, \mathrm{Q}_{2} \in \mathcal{P}$ be given, such that

(C1) $\overrightarrow{\mathrm{P}}_{1}, \overrightarrow{\mathrm{P}}_{2} \in \vec{\Gamma}(\mathrm{x}, \mathrm{M})$ with $\vec{\Gamma}(\mathrm{x}, \mathrm{M})$ as in (2.10);

(C2) $\left\|\partial^{\alpha}\left(\vec{P}_{1}-\vec{P}_{2}\right)(x)\right\|_{\infty} \leq M \delta^{m-|\alpha|}$ for $|\alpha| \leq m-1$;

(C3) $\left|\partial^{\alpha} Q_{i}(x)\right| \leq \delta^{-|\alpha|}$ for $|\alpha| \leq m-1, i=1,2$; and

(C4) $\mathrm{Q}_{1} \odot_{x} \mathrm{Q}_{1}+\mathrm{Q}_{2} \odot_{x} \mathrm{Q}_{2}=1$.

We set

$$
\overrightarrow{\mathrm{P}}:=\sum_{i=1,2} \mathrm{Q}_{i} \odot_{x} \mathrm{Q}_{i} \odot_{\chi} \overrightarrow{\mathrm{P}}_{i} .
$$

We want to show that $\vec{P} \in \vec{\Gamma}(x, C M)$ for some $C=C(m, n, D)$.

It is clear from $(\mathrm{C} 1)$ and $(\mathrm{C} 4)$ that $\overrightarrow{\mathrm{P}}(\mathrm{x})=\mathrm{K}(\mathrm{x})$. It remains to show that $\left\|\partial^{\alpha} \vec{P}(x)\right\|_{\infty} \leq \mathrm{CM}$.

Using the product rule, we have, for $|\alpha| \leq m-1$,

$$
\partial^{\alpha} \vec{P}(x)=\sum_{i=1,2} \sum_{\beta \leq \alpha} \sum_{\gamma \leq \beta} C_{\alpha, \beta, \gamma} \cdot \partial^{\gamma} Q_{i}(x) \cdot \partial^{\beta-\gamma} Q_{i}(x) \cdot \partial^{\alpha-\beta} \vec{P}_{i}(x) .
$$

By (C4), we have $\partial^{\alpha}\left(\mathrm{Q}_{2} \odot_{\chi} \mathrm{Q}_{2}\right)=-\partial^{\alpha}\left(\mathrm{Q}_{1} \odot_{\chi} \mathrm{Q}_{1}\right)$ for $|\alpha|>0$. It follows from $(\mathrm{C} 2)$ and (C3) that $\left\|\partial^{\alpha} \vec{P}(x)\right\|_{\infty} \leq \mathrm{CM}$.

Thus, it remains to establish Theorem 1.4 This will be done in Section 4 


\section{WHITNEY NORM AND DUAL NORM ON CLUSTERS}

In this section, we review the data structure in [1], and prove a series of results that allows us to reduce the size of supports for linear functionals on $W^{m}(S)^{*}$.

We write $|S|$ to denote the cardinality of a finite set $S \subset \mathbb{R}^{n}$.

If $X, Y \subset \mathbb{R}^{n}$, we define

$$
\begin{aligned}
\operatorname{diam}(X) & :=\max _{x, x^{\prime} \in X}\left|x-x^{\prime}\right| \text { and } \\
\operatorname{dist}(X, Y) & :=\min _{x \in X, y \in Y}|x-y| .
\end{aligned}
$$

A rooted tree ("tree" for short) is an undirected graph with a distinct node (i.e., the root) in which any two nodes are connected by exactly one path. A leaf of a tree is any non-root node of degree one.

Let $S \subset \mathbb{R}^{n}$ be a finite set. We consider trees $\mathcal{T}$, each node of which corresponds to a subset of $S$, that satisfy the following properties.

(T1) The root of $\mathcal{T}, \mathrm{R}(\mathcal{T})=\mathrm{S}$.

(T2) If $\mathrm{V}$ is a node, then $\mathrm{V}$ corresponds to a subset of $\mathrm{S}$. The children of any node $\mathrm{V}$ form a partition of $\mathrm{V}$ (unless $\mathrm{V}$ is a leaf).

(T3) The nodes of any given level correspond to a partition of $\mathrm{S}$. The leaves of $\mathcal{T}$ are the singletons $\{x\}$, with $x \in S$.

(T4) The number of nodes of level $\ell=0,1, \ldots$ is a strictly increasing function of $\ell$.

A collection of points

$$
\underline{x}=\left\{x_{\vee} \in S: V \in \mathcal{T} \backslash \operatorname{leaves}(\mathcal{T})\right\}
$$

is called a set of reference points for $\mathcal{T}$ if, for each $V, x_{V} \in V$ and $x_{V}=x_{W}$ for

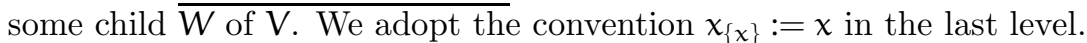

Let $\underline{x}$ be a set of reference points of $\mathcal{T}$. For each $V \in \mathcal{T} \backslash$ leaves $(\mathcal{T})$, define

$$
\mathrm{V}(\underline{\mathrm{x}}):=\left\{x_{\mathrm{W}}: \mathrm{W} \text { is a child of } \mathrm{V}\right\} \text {. }
$$

Suppose $x \in S \backslash\left\{x_{S}\right\}$. Then there is a unique node $V$ of highest level such that $x \in V \backslash\left\{x_{V}\right\}$. We set

$$
\operatorname{ref}(x):=x_{V}
$$

We also set

$$
\mathrm{U}(x):=\text { the oldest ancestor of } \mathrm{U} \text { such that } x=\chi_{\mathrm{U}} \text {. }
$$

A trunk $T$ of $\mathcal{T}$ denotes a directed path from the root $S$ to level height $(\mathcal{T})-1$. Let $T$ be a trunk of $\mathcal{T}$. We define the set of branch nodes $B(T)$ as the set of nodes of $\mathcal{T}$ which are adjacent to $T$.

Recall that the height of a tree is defined to be the number of edges on the longest downward path between the root and a leaf. We define the notion of "clustering" as follows.

Definition 3.1. Let $\mathrm{S} \subset \mathbb{R}^{n}$ be finite. Let $\mathcal{T}$ be a tree of subsets of $\mathrm{S}$ that satisfies (T1) to (T4). We say that $\mathcal{T}$ is a clustering of $\mathrm{S}$ if $\mathcal{T}$ has a set of reference points $\underline{x}=\left\{\chi_{\mathrm{V}}\right\}$ such that for each $\mathrm{l}=0, \overline{1, \cdots, \text { height }}(\mathcal{T})-1$, the set

$$
\Pi:=\{\mathrm{V}(\underline{x}): \operatorname{level}(\mathrm{V})=\ell\}
$$

forms a partition of

$$
\left\{a_{W}: \operatorname{level}(W)=\ell+1\right\}
$$


satisfying

$$
\begin{aligned}
|x-y| & \geq c_{\underline{x}} \cdot \operatorname{diam}(S) \text { for each } S \in \Pi, x \neq y \text { in } S \text {, and } \\
\operatorname{dist}\left(S, S^{\prime}\right) & \geq \underline{c}_{\underline{x}} \cdot \operatorname{diam}(S) \text { for all } S, S^{\prime} \in \Pi, S \neq S^{\prime} .
\end{aligned}
$$

Here, $0<\mathrm{c}_{\underline{x}}(\mathrm{n},|\mathrm{S}|) \leq 1$ is called the clustering constant.

We write $\mathcal{C}=\mathcal{C}(\mathcal{T}, \underline{x})$ to denote a clustering $\mathcal{T}$ of $S$ together with a set of reference points $\underline{x}$.

Lemma 3.2 (Lemma 2.4 of [1]). Given a finite set $S \subset \mathbb{R}^{n}$, we can always find a clustering $\mathcal{T}$ of $\mathbf{S}$ such that for any set of reference points $\underline{x}$ for $\mathcal{T}$, the condition of Definition 3.1 is satisfied with some $0<\mathrm{c}_{\underline{x}} \leq 1$, where $\mathrm{c}_{\underline{\underline{x}}}$ depends only on $\mathrm{m}, \mathrm{n}$, and \#(S).

Definition 3.3. Let $\mathcal{C}=\mathcal{C}(\mathcal{T}, \underline{x})$ be a clustering of $\mathrm{S}$ with a set of reference points $\underline{\mathrm{x}}$. We define the $\mathrm{C}^{\mathrm{m}}$-clustering norm $\|\cdot\|_{\mathcal{C}}$ on $\mathrm{W}^{\mathrm{m}}(\mathrm{S})$ to be

$$
\left\|\left(\overrightarrow{\mathrm{P}}^{x}\right)_{x \in S}\right\|_{\mathcal{C}}:=\max \left\{\left\|\left(\overrightarrow{\mathrm{P}}^{x}\right)_{x \in S}\right\|_{\mathcal{C}},\left\|\overrightarrow{\mathrm{P}}^{x_{S}}\right\|_{x_{S}}\right\},
$$

where

$$
\|\overrightarrow{\mathrm{P}}\| \|_{\mathcal{C}}:=\max _{\substack{x \in S \backslash\left\{x_{S}\right\} \\ y=r e f(x) \\|\alpha| \leq m}} \frac{\left\|\partial^{\alpha}\left(\overrightarrow{\mathrm{P}}^{\mathrm{x}}-\overrightarrow{\mathrm{P}}^{y}\right)(x)\right\|_{\infty}}{|x-y|^{m-|\alpha|}} \text { and }\left\|\overrightarrow{\mathrm{P}}^{x_{S}}\right\|_{x_{S}}:=\max _{|\alpha| \leq \mathrm{m}}\left\|\partial^{\alpha} \overrightarrow{\mathrm{P}}^{x_{S}}\left(x_{S}\right)\right\|_{\infty}
$$

Lemma 3.4 (Proposition 3.2 of [1]). Let $\mathrm{S} \subset \mathbb{R}^{\mathrm{n}}$ be a finite set, and let $\mathcal{C}=\mathcal{C}(\mathcal{T}, \underline{\mathrm{x}})$ be a clustering of $\mathbf{S}$ with a set of reference points $\underline{\mathbf{x}}$ and clustering constant $\mathbf{c}_{\underline{\mathbf{x}}}$. Then

$$
\left\|\left(\overrightarrow{\mathrm{P}}^{\mathrm{x}}\right)_{x \in S}\right\|_{W^{\mathrm{m}}(\mathrm{S})} \leq \mathrm{C}\left\|\left(\overrightarrow{\mathrm{P}}^{\mathrm{x}}\right)_{x \in S}\right\|_{\mathcal{C}} .
$$

Here, $\mathrm{C}=\mathrm{C}\left(\mathrm{c}_{\underline{x}}, \mathrm{~m}, \mathrm{n},|\mathrm{S}|, \mathrm{B}\right)$, where $\mathrm{B}$ is an upper bound on $\operatorname{diam}(\mathrm{S})$.

Next we characterize linear functionals on clusters.

Let $S \subset \mathbb{R}^{n}$ be finite, and let $\xi=\left(\xi_{x}\right)_{x \in S} \in W^{m}(S)^{*}$.

Let $\mathcal{C}(\mathcal{T}, \underline{x})$ be a clustering of $S$. For each node $V \in \mathcal{T}$, we define $\xi_{V}$ by the formula

$$
\xi_{\mathrm{V}}:=\sum_{x \in \mathrm{V}} \xi_{x} .
$$

Lemma 3.5 (Lemma 5.1 of [1]). Let $\mathrm{S} \subset \mathbb{R}^{n}$ be a finite set, and let $\mathcal{C}=\mathcal{C}(\mathcal{T}, \underline{x})$ be a clustering of $\mathbf{S}$ with a set of reference points $\underline{\mathbf{x}}$ and clustering constant $\mathbf{c}_{\mathbf{x}}$. Let ref $(\mathrm{x})$ and $\mathrm{U}(\mathrm{x})$ be as in (3.1) and (3.2), respectively. The action of $\xi \in \mathrm{W}^{\mathrm{m}}(\mathrm{S})^{*}$ has the form:

$$
\xi\left[\left(\overrightarrow{\mathrm{P}}^{x}\right)_{x \in S}\right]=\sum_{x \in S \backslash\left\{x_{S}\right\}} \xi_{u(x)}\left(\overrightarrow{\mathrm{P}}^{x}-\overrightarrow{\mathrm{P}}^{\mathrm{ref}(\mathrm{x})}\right)+\xi_{S}\left(\overrightarrow{\mathrm{P}}^{x_{S}}\right) .
$$

As a consequence, we can compute the cluster dual norm using the formula:

$$
\begin{aligned}
\|\xi\|_{\mathcal{C}^{*}}= & \sum_{\substack{x \in S \backslash\left\{x_{S}\right\} \\
|\alpha| \leq m \\
1 \leq j \leq \mathrm{D}}}|x-\operatorname{ref}(x)|^{m-|\alpha|}\left|\xi_{\mathrm{u}(x)}\left(0, \ldots, 0, \frac{(\cdot-x)^{\alpha}}{\alpha !}, 0, \ldots, 0\right)\right| \\
& +\sum_{\substack{|\alpha| \leq \mathrm{m} \\
1 \leq j \leq \mathrm{D}}}\left|\xi_{S}\left(0, \ldots, 0, \frac{\left(\cdot-x_{S}\right)^{\alpha}}{\alpha !}, 0, \ldots, 0\right)\right| .
\end{aligned}
$$


In the above, the nontrivial expression in the arguments of $\xi_{\mathrm{s}}$ and $\xi_{\mathrm{u}(\mathrm{x})}$ are in the $j$-th coordinates.

Lemma 3.6. Let $S \subset \mathbb{R}^{n}$ be a finite set, and let $\Phi: S \times \overrightarrow{\mathcal{P}}^{*} \rightarrow \mathbb{R}$ be a function that is positively homogeneous with degree one on the fibers and vanishes along the zero section. Let $\mathcal{T}$ be a clustering of of $\mathrm{S}$. Let $\xi \in \mathrm{W}^{\mathrm{m}}(\mathrm{S})^{*}$. For each $\mathrm{V} \in \mathcal{T}$, define $\xi_{\mathrm{V}}$ as in (3.5). Define

$$
\Phi\left(\xi_{\mathrm{V}}\right):=\sum_{\mathrm{x} \in \mathrm{V}} \Phi_{\mathrm{x}}\left(\xi_{\mathrm{x}}\right)
$$

and set $\bar{\xi}_{\mathrm{V}}:=\left(\xi_{\mathrm{V}}, \Phi\left(\xi_{\mathrm{V}}\right)\right) \in \overrightarrow{\mathcal{P}}^{*} \oplus \mathbb{R}$. Let $\mathrm{T}$ be a trunk of $\mathcal{T}$, and let $\Xi(\mathrm{T})$ denote the linear span of $\left\{\bar{\xi}_{V}: \mathrm{V} \in \mathrm{B}(\mathrm{T})\right\}$ in $\overrightarrow{\mathcal{P}}^{*} \oplus \mathbb{R}$. Assume

$$
\operatorname{dim} \Xi(\mathrm{T})<\#(\mathrm{~B}(\mathrm{~T})) \text {. }
$$

Then there exists $\eta \in W^{m}(S)^{*}$ such that the following hold.

(1) For all $\mathrm{V} \in \mathcal{T} \backslash \mathrm{T}, \eta_{\vee}=\theta_{\mathrm{V}} \xi_{\mathrm{V}}$ for some $0 \leq \theta_{\mathrm{V}} \leq 2$

(2) For some $\mathrm{V} \in \mathrm{B}(\mathrm{T}), \eta_{\mathrm{x}} \equiv 0$ for all $\mathrm{x} \in \mathrm{V}$.

(3) $\sum_{x \in S} \xi_{x}=\sum_{x \in S} \eta_{x}$ as elements of $\overrightarrow{\mathcal{P}}^{*}$.

(4) $\sum_{x \in S} \Phi_{x}\left(\xi_{x}\right)=\sum_{x \in S} \Phi_{x}\left(\eta_{x}\right)$.

Moreover, for such $\eta$, we have

$$
\|\eta\|_{\mathcal{C}^{*}} \leq 2\|\eta\|_{\mathcal{C}^{*}}
$$

Proof. We modify the proof of Lemma 6.1 of 1 .

Since $\operatorname{dim} \Xi(T) \leq \#(B(T)),\left\{\bar{\varepsilon}_{W}, W \in B(T)\right\}$ is not linearly independent, so we may find $V \in B(T)$ such that

$$
\bar{\xi}_{V}=\sum_{W \in B(T) \backslash V} \lambda_{V W} \cdot \bar{\xi}_{W} \text { where all }\left|\lambda_{V W}\right| \leq 1 .
$$

For each $x \in S$, we set $\eta_{x}:=\theta_{x} \cdot \xi_{x}$, where

$$
\theta_{x}:= \begin{cases}0 & \text { if } x \in V . \\ 1+\lambda_{V W} & \text { if } x \in W \text { and } W \in B(T) \backslash V . \\ 1 & \text { otherwise. }\end{cases}
$$

Conditions (1) and (2) then follow by construction.

Now we prove (3) and (4). First we make the following crucial observation. Thanks to our assumption on $\Phi$ and the conditions on the $\lambda_{V w}$ 's, we see that

$$
\Phi_{x}\left(\left(1+\lambda_{V w}\right) \xi_{x}\right)=\Phi_{x}\left(\xi_{x}\right)+\lambda_{V w} \Phi_{x}\left(\xi_{x}\right) .
$$

Therefore,

$$
\begin{aligned}
\sum_{x \in S} \bar{\eta}_{x} & =\sum_{x \in S} \bar{\xi}_{x}-\sum_{x \in V} \bar{\xi}_{x}+\sum_{W \in \mathrm{B}(\mathrm{T}) \backslash \mathrm{V}} \lambda_{\mathrm{V} W} \sum_{x \in W} \bar{\xi}_{x} \\
& =\sum_{x \in S} \bar{\xi}_{x}-\left(\bar{\xi}_{V}-\sum_{W \in \mathrm{B}(\mathrm{T}) \backslash \mathrm{V}} \lambda_{\mathrm{V} W} \bar{\xi}_{W}\right) \\
& =\sum_{x \in S} \bar{\xi}_{x} .
\end{aligned}
$$

We see that (3) and (4) follow.

Lastly, (3.8) follows from (3.7) and conditions (1) and (3). 
Let $S \subset \mathbb{R}^{n}$ and let $\mathcal{T}$ be a clustering of $S$. For any subset $S^{\prime} \subset S, \mathcal{T}$ determines a clustering $\mathcal{T}^{\prime}$ of $S^{\prime}$ by restriction.

The main result of the section is the following.

Lemma 3.7. Let $\mathrm{k} \geq 2$. Under the hypotheses of Lemma 3.6, if $|\mathrm{S}| \leq \mathrm{k}$, then there exists $S^{\prime} \subset S$ satisfying the following.

(1) Let $\mathcal{T}^{\prime}$ be the clustering of $\mathrm{S}^{\prime}$ determined by $\mathcal{T}$. For every trunk $\mathrm{T}^{\prime}$ of $\mathcal{T}^{\prime}$, let $\Xi\left(\mathrm{T}^{\prime}\right)$ denote the linear span of $\left\{\bar{\xi}_{\mathrm{V}}: \mathrm{V} \in \mathrm{B}\left(\mathrm{T}^{\prime}\right)\right\}$ in $\overrightarrow{\mathcal{P}}^{*} \oplus \mathbb{R}$. Then we have

$$
\#\left(B\left(\mathrm{~T}^{\prime}\right)\right) \leq \operatorname{dim} \Xi\left(\mathrm{T}^{\prime}\right) \text {. }
$$

(2) There exists $\eta \in \mathrm{W}^{\mathrm{m}}(\mathrm{S})^{*}$ such that the following hold.

(a) $\eta_{x}$ is a multiple of $\xi_{x}$ for each $x \in S$, and $\eta_{x}=0$ for $x \in S \backslash S^{\prime}$.

(b) $\|\eta\|_{W^{m}(S)^{*}} \leq \mathrm{C}\|\xi\|_{W^{m}(S)^{*}}$, where $\mathrm{C}=\mathrm{C}(\mathrm{m}, \mathrm{n}, \mathrm{k}, \mathrm{B})$ with $\mathrm{B}$ being an upper bound for $\operatorname{diam}(\mathrm{S})$.

(c) $\sum_{x \in S} \xi_{x}=\sum_{x \in S} \eta_{x}$.

(d) $\sum_{x \in S}^{x \in S} \Phi_{x}\left(\xi_{x}\right) \stackrel{x \in S}{=} \sum_{x \in S} \Phi_{x}\left(\eta_{x}\right)$, with $\Phi_{x}$ as in Lemma 3.6.

Proof. Suppose $S$ itself does not satisfy both of the conclusions. Taking $\eta=\xi$, we see that $\mathbf{S}$ satisfies (2). Therefore, $S$ does not satisfy (1). Using condition (2) of Lemma 3.6 we may shrink $S$ by one point at a time until conclusions (1), (2a), (2c), and (2d) hold. Meanwhile, (2b) holds, thanks to Lemma 3.2 Lemma 3.4, and (3.8).

We will couple Lemma 3.7 with the following result.

Lemma 3.8 (Lemma 6.4 of [1]). Let $\mathrm{S} \subset \mathbb{R}^{n}$ be finite with $\#(\mathrm{~S}) \geq 2$. Let $\mathcal{T}$ be a clustering of $\mathrm{S}$. Suppose that for every trunk $\mathrm{T}$ of $\mathcal{T},|(\mathrm{B}(\mathrm{T}))| \leq \mathrm{N}$ for some $\mathrm{N} \in \mathbb{N}$. Then $|\mathrm{S}| \leq 2^{\mathrm{N}-1}$.

\section{Proof of the Main theorem}

We begin the proof of Theorem 1.4 by showing that one can approximate the convex sets $\Gamma$ arbitrarily well by polytopes, which will allow us to use linear programming. While finer levels of approximation to these convex sets will generally require an arbitrarily increasing number of linear constraints to describe, the constants arising in our proof will be independent of this number.

By a polytope in a finite-dimensional normed vector space $V$, we mean the finite intersection of half-spaces of the form $\{v \in \mathrm{V}: \xi(v) \leq \mathrm{c}\}$, where $\xi \in \mathrm{V}^{*}$ and $\mathrm{c} \in \mathbb{R}$.

Let $v, w$ be two Euclidean vectors. We write $v \geq w$ if each of the entries of $v-w$ is nonnegative.

Lemma 4.1. Let $\mathrm{V}$ be a finite-dimensional normed vector space with norm $\|\cdot\|_{\mathrm{V}}$, and let $\mathrm{K} \subset \mathrm{V}$ be convex. Given $\delta>0$, there exists a convex polytope $\mathrm{K}_{\delta}$ such that $\mathrm{K} \subset \mathrm{K}_{\delta} \subset \mathrm{B}_{\delta}(\mathrm{K})$, where $\mathrm{B}_{\delta}(\mathrm{K})$ is the $\delta$-neighborhood of $\mathrm{K}$ under the metric determined by $\|\cdot\|_{V}$.

Proof. We first address the case where $\mathrm{V}=\mathbb{R}^{\mathrm{d}}$, where the norm is the $\ell^{\infty}$ norm given by $\left\|\left(x_{1}, \ldots, x_{d}\right)\right\|=\max _{1 \leq j \leq d}\left|x_{j}\right|$.

Let $\mathcal{Q}$ be the set of cubes of the form

$$
\mathrm{Q}=\left[\mathrm{k}_{1} \delta,\left(\mathrm{k}_{1}+1\right) \delta\right] \times \ldots \times\left[\mathrm{k}_{\mathrm{d}} \delta,\left(\mathrm{k}_{\mathrm{d}}+1\right) \delta\right],
$$


where $k_{1}, \ldots, k_{d} \in \mathbb{Z}$. Define

$$
\mathrm{K}^{\prime}=\bigcup_{\substack{\mathrm{Q} \in \mathcal{Q} \\ \mathrm{Q} \cap \mathrm{K} \neq \emptyset}} \mathrm{Q},
$$

and let $K^{\prime \prime}=\operatorname{Conv}\left(K^{\prime}\right)$, where $\operatorname{Conv}(\cdot)$ is used to denote the convex hull of a set. Thus, $K^{\prime \prime}$ is a convex polytope. By definition, $K \subset K^{\prime}$.

Let $x \in K^{\prime \prime}$. Then, there exist $y^{\prime}, z^{\prime} \in K^{\prime}$ such that $x$ is on the line segment from $y^{\prime}$ to $z^{\prime}$. Since $y^{\prime}, z^{\prime} \in K^{\prime}$, there exist $y, z \in K$ such that $\left\|y-y^{\prime}\right\|,\left\|z-z^{\prime}\right\|<\delta$.

Consider the function $f(t)=\left\|t\left(y^{\prime}-y\right)+(1-t)\left(z^{\prime}-z\right)\right\|$. Then $f(0), f(1)<\delta$ and $f$ is a convex, nonnegative function, so $f(t)<\delta$ for all $t \in[0,1]$. Pick $t_{0} \in[0,1]$ such that $x=t_{0} y^{\prime}+\left(1-t_{0}\right) z^{\prime}$. Then, $f\left(t_{0}\right)<\delta$ means that $\left\|x-\left[t y+(1-t) y^{\prime}\right]\right\|<\delta$. Since $K$ is convex, $t y+(1-t) y^{\prime} \in K$, so $x$ is within distance $\delta$ of $K$. Thus, $K^{\prime} \subset B_{\delta}(K)$, completing the proof in the case $\mathrm{V}=\mathbb{R}^{\mathrm{d}}$.

Now suppose that $V$ is an arbitrary d-dimensional, normed space. Since any two norms on a finite-dimensional space are equivalent, there exists $M<\infty$ such that $\mathrm{M}^{-1}\|v\|_{V} \leq\left\|\mathrm{T}^{-1}(v)\right\|_{\ell^{\infty}\left(\mathbb{R}^{\mathrm{d}}\right)} \leq \mathrm{M}\|v\|_{V}$.

Let $\mathrm{T}: \mathbb{R}^{\mathrm{d}} \rightarrow \mathrm{V}$ be a linear isomorphism and let $\tilde{\mathrm{K}} \subset \mathbb{R}^{\mathrm{n}}$ be a polytope satisfying $\mathrm{T}^{-1}(\mathrm{~K}) \subset \tilde{\mathrm{K}} \subset \mathrm{B}_{\epsilon}\left(\mathrm{T}^{-1}(\mathrm{~K})\right)$, where $\epsilon>0$ is to be determined. It follows that $\mathrm{K} \subset \mathrm{T}(\tilde{\mathrm{K}}) \subset \mathrm{B}_{\mathrm{M \epsilon}}(\mathrm{K})$ and that $\mathrm{T}(\tilde{\mathrm{K}})$ is a polytope in $\mathrm{V}$. (To see the latter, observe that for linear functionals $\xi$ on $\mathbb{R}^{\mathrm{d}}, \xi(v) \leq \mathrm{c}$ if and only if $\xi \circ \mathrm{T}^{-1}(\mathrm{~T}(v)) \leq \mathrm{c}$ and $\xi \circ \mathrm{T}^{-1} \in \mathrm{V}^{*}$.)

Thus, choosing $\epsilon=\delta / M$, we see that $T(\tilde{K})$ is the desired polytope.

4.1. Theorem 1.4 with $\mathbb{X}=\mathrm{C}^{\mathrm{m}}\left(\mathbb{R}^{\mathrm{n}}, \mathbb{R}^{\mathrm{D}}\right)$.

Proof of Theorem 1.4 with $\mathbb{X}=\mathrm{C}^{\mathrm{m}}\left(\mathbb{R}^{n}, \mathbb{R}^{\mathrm{D}}\right)$. Given $\mathrm{E} \subset \mathbb{R}^{n}$ and $\mathrm{K}(\mathrm{x}) \subset \overrightarrow{\mathcal{P}}$ for each $x \in E$, we define

$$
\left\|(K(x))_{x \in E}\right\|_{W^{m}(E)}:=\inf \left\{\left\|\left(\vec{P}^{x}\right)_{x \in E}\right\|_{W^{m}(E)}: \vec{P}^{x} \in K(x) \text { for all } x \in E\right\} .
$$

While not strictly a norm, the above notation allows for a concise description of a quantity which is the main focus of the proof.

Our goal is to show there exists $C=C(m, n, D, B)$ such that for any finite $S \subset \mathbb{R}^{n}$ satisfying $|S| \leq B$, there exists $S^{\prime} \subset S$ with $\left|S^{\prime}\right| \leq 2^{\operatorname{dim} \overrightarrow{\mathcal{P}}}$ such that

$$
\mathrm{C}^{-1}\left\|(\mathrm{G}(\mathrm{x}))_{\mathrm{x} \in \mathrm{S}^{\prime}}\right\|_{\mathrm{W}^{\mathrm{m}}\left(\mathrm{S}^{\prime}\right)} \leq\left\|(\mathrm{G}(\mathrm{x}))_{\mathrm{x} \in \mathrm{S}}\right\|_{\mathrm{W}^{\mathrm{m}}(\mathrm{S})} \leq \mathrm{C}\left\|(\mathrm{G}(\mathrm{x}))_{\mathrm{x} \in \mathrm{S}^{\prime}}\right\|_{W^{\mathrm{m}}\left(\mathrm{S}^{\prime}\right)} .
$$

If so, it follows that

$$
\left\|(G(x))_{x \in S^{\prime}}\right\|_{W^{m}\left(S^{\prime}\right)} \leq 1 \text { for all } S^{\prime} \subset S \text { satisfying }\left|S^{\prime}\right|=2^{\operatorname{dim} \overrightarrow{\mathcal{P}}}
$$

implies

$$
\left\|(\mathrm{G}(\mathrm{x}))_{\mathrm{x} \in \mathrm{S}}\right\|_{W^{\mathrm{m}}(\mathrm{S})} \leq \mathrm{C}
$$

for all $S \subset E$ satisfying $|S|=k^{\sharp}$.

We now make the following reduction: it suffices to prove Theorem 1.4 in the case that each $G(x)$ is a polytope.

If not, replace each $G(x)$ with $G(x)_{\delta}$ for sufficiently small $\delta>0$, where $G(x)_{\delta}$ is the polytope guaranteed by Lemma 4.1. By taking $\delta>0$ small enough, one may approximate both $\left\|(G(x))_{x \in S}\right\|_{W^{m}(S)}$ and $\left\|(G(x))_{x \in S^{\prime}}\right\|_{W^{m}\left(S^{\prime}\right)}$ within a factor of 2 , as these norms are continuous with respect to the relevant metrics.

To this end, we replace each $G(x)$ with $G(x)_{\delta}$, which will now be denoted $K_{x}$, as $\delta$ is fixed. For each $x$, we write $K_{\chi}=\left\{\vec{P}: \Omega_{\chi} \vec{P} \leq \overrightarrow{\mathcal{c}}_{\chi}\right\}$ for some linear map 
$\Omega_{x}: \overrightarrow{\mathcal{P}} \rightarrow \mathbb{R}^{m_{x}}$, where $\mathrm{m}_{x} \in \mathbb{N}$. We will occasionally write $\Omega: \mathrm{W}^{\mathrm{m}}(\mathrm{S}) \rightarrow \prod_{\chi} \mathbb{R}^{\mathrm{m}_{\mathrm{x}}}$ to denote the mapping which sends $\left(\overrightarrow{\mathrm{P}}^{x}\right)_{x \in S}$ to $\left(\Omega_{x} \overrightarrow{\mathrm{P}}^{x}\right)_{x \in S}$.

We begin by writing $\left\|\left(K_{x}\right)_{x \in S}\right\|_{W^{m}(S)}$ as the solution to a linear programming problem:

$$
\begin{aligned}
& \left\|\left(K_{x}\right)_{x \in S}\right\|_{W^{m}(S)}=\inf _{\left(\Omega_{x} \vec{P}^{x} \leq \vec{c}_{x}\right)_{x \in S}}\left\|\left(\vec{P}^{x}\right)_{x \in S}\right\|_{W^{m}(S)}
\end{aligned}
$$

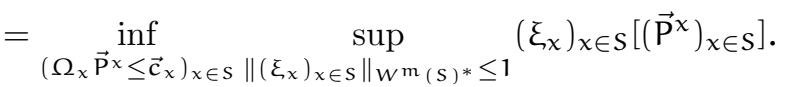

By Lemma 4.1, the unit ball in $\mathrm{W}^{\mathrm{m}}(\mathrm{S})^{*}$ may be approximated within a factor of 2 by a polytope, written as $\left\{\left(\xi_{x}\right)_{x \in S}: L\left(\xi_{x}\right)_{x \in S} \leq \mathbf{1}_{k}\right.$ for some $k \in \mathbb{N}$ and linear map $L: W^{m}(S)^{*} \rightarrow \mathbb{R}^{k}$. Thus, we may rewrite (4.2) as

$$
\left\|\left(K_{x}\right)_{x \in S}\right\|_{W^{m}(S)} \approx \inf _{\left(\Omega_{x} \vec{P}^{x} \leq \vec{c}_{x}\right)_{x \in S} L\left(\xi_{x}\right)_{x \in S} \leq 1_{k}}\left(\xi_{x}\right)_{x \in S}\left[\left(\vec{P}^{x}\right)_{x \in S}\right]
$$

for some linear map $L: W^{m}(S)^{*} \rightarrow \mathbb{R}^{k}$ and some $k \in \mathbb{N}$.

The advantage of this formulation is that it becomes possible to apply the LP Duality Theorem (Lemma A.2 in Appendix) to the supremum above, giving us

$$
\begin{aligned}
\left\|\left(K_{x}\right)_{x \in S}\right\|_{W^{m}(S)} \approx \inf _{\left(\Omega_{x} \vec{P}^{x} \leq \overrightarrow{\mathbf{c}}_{x}\right)_{x \in S}} \inf _{\substack{y \geq 0 \\
L^{\top} y=\left(\overrightarrow{\mathrm{P}}^{x}\right)_{x \in S}}} \mathbf{1}_{k} \cdot y \\
=\inf _{\substack{\left(\Omega_{x} \overrightarrow{\mathrm{P}}^{x} \leq \mathbf{c}_{x}\right)_{x \in S} \\
y \geq 0}} \mathbf{1}_{k} \cdot y \\
=\inf _{\substack{y \geq 0 \\
L^{\top} y=\left(\overrightarrow{\mathrm{P}}^{\mathrm{x}}\right)_{x \in S} \\
y \geq 0}} \mathbf{1}_{k} \cdot y .
\end{aligned}
$$

Note the referenced linear programming problem is feasible, as its solution corresponds to finding the smallest norm of a vector in a closed set.

Applying the Duality Theorem again, one obtains

$$
\begin{aligned}
\left\|\left(\mathrm{K}_{x}\right)_{x \in S}\right\|_{W^{\mathrm{m}}(\mathrm{S})} \approx & \sup _{\substack{\left(z_{x} \geq 0\right)_{x \in S} \\
\mathrm{~L}\left(-\Omega^{\mathrm{T}}\right) z \leq \mathbf{1}_{\mathrm{k}}}} \sum_{x}-\overrightarrow{\mathcal{c}}_{x} \cdot z_{x} \\
= & \sup _{\substack{\mathrm{L}\left(\xi_{x}\right)_{x} \in S \leq \mathbf{1}_{\mathrm{k}} \\
\left(\xi_{x} \in\left(-\Omega_{x}^{\top}\right) \mathbb{R}_{+}^{m_{x}}\right)_{x \in S}}} \sup _{\substack{\left(z_{x} \geq 0\right)_{x \in S} \\
\left(-\Omega_{x}^{\top} z_{x}=\xi_{x}\right)_{x \in S}}} \sum_{x}-\overrightarrow{\mathcal{c}}_{x} \cdot z_{x} \\
& \approx \sup _{\left(\xi_{x} \in\left(-\Omega_{x}^{\mathrm{T}}\right) \mathbb{R}_{+}^{m_{x}}\right)_{x \in S}} \frac{\sum_{x \in S} f_{x}\left(\xi_{x}\right)}{\left\|\left(\xi_{x}\right)_{x \in S}\right\|_{W^{m}(S)^{*}}},
\end{aligned}
$$

where

$$
f_{x}\left(\xi_{x}\right)=\sup _{\substack{z \geq 0 \\-\Omega_{x}^{\top} z=\xi_{x}}} \sum_{x}-\vec{c}_{x} \cdot z .
$$

Fix $\left(\xi_{x}\right)_{x \in S}$ such that $\xi_{x} \in\left(-\Omega_{x}^{\top}\right) \mathbb{R}_{+}^{m_{x}}$ for all $x \in S$. We see that $f$ satisfies the hypotheses of Lemmas 3.6 and 3.7. By Lemma 3.8 we may apply Lemma 3.7 repeatedly until the $S^{\prime}$ in the conclusion satisfies $\left|S^{\prime}\right| \leq 2^{\operatorname{dim} \mathcal{P}}$. 
Let $\left(\eta_{x}\right)_{x \in S}$ be as guaranteed in the conclusion of the lemma and recall $S^{\prime}=$ $\left\{x \in S: \eta_{x} \neq 0\right\}$. Thus,

$$
\left\|\left(\eta_{x}\right)_{x \in S}\right\|_{W^{m}\left(S^{\prime}\right)^{*}}=\left\|\left(\eta_{x}\right)_{x \in S}\right\|_{W^{m}(S)^{*}} \lesssim\left\|\left(\xi_{x}\right)_{x \in S}\right\|_{W^{m}(S)^{*}}
$$

and $\left|S^{\prime}\right| \leq 2^{\operatorname{dim} \overrightarrow{\mathcal{P}}}$. Note that each $\eta_{x}$ is obtained by multiplying some $\xi_{x}$ by a nonnegative scalar; thus, $\eta_{x} \in\left(-\Omega_{x}^{\top}\right) \mathbb{R}_{+}^{m_{x}}$ for all $x \in S$.

By this reasoning and (4.5) applied both as written above and with $S^{\prime}$ in place of $\mathrm{S}$,

$$
\begin{aligned}
\left\|\left(K_{x}\right)_{x \in S}\right\|_{W^{m}(S)} & \approx \sup _{\left(\xi_{x} \in \Omega_{x}^{\top} \mathbb{R}_{+}^{m_{x}}\right)_{x \in S}} \frac{\sum_{x \in S} f_{x}\left(\xi_{x}\right)}{\left\|\left(\xi_{x}\right)_{x \in S}\right\|_{W^{m}(S)^{*}}} \\
& \lesssim \sup _{\left(\eta_{x} \in \Omega_{x}^{\top} \mathbb{R}_{+}^{m_{x}}\right)_{x \in S^{\prime}}} \frac{\sum_{x \in S} f_{x}\left(\eta_{x}\right)}{\left\|\left(\eta_{x}\right)_{x \in S}\right\|_{W^{m}\left(S^{\prime}\right)^{*}}} \\
& \approx\left\|\left(K_{x}\right)_{x \in S^{\prime}}\right\|_{W^{m}\left(S^{\prime}\right) .}
\end{aligned}
$$

4.2. Theorem 1.4 with $\mathbb{X}=\dot{\mathrm{C}}^{\mathrm{m}}\left(\mathbb{R}^{\mathrm{n}}, \mathbb{R}^{\mathrm{D}}\right)$. In this section, we point out the modifications needed in order to prove Theorem 1.4 for the case $\mathbb{X}=\dot{\mathrm{C}}^{\mathrm{m}}\left(\mathbb{R}^{\mathrm{n}}, \mathbb{R}^{\mathrm{D}}\right)$.

Let $S \subset \mathbb{R}^{n}$ be a finite set. Recall the definition of $\|\cdot\|_{\dot{W}^{m}(S)}$ in (2.2). We define

$$
\mathrm{H}(\mathrm{S}):=\operatorname{span}\left\{\xi_{\alpha, y, z, j}: y, z \in S, z \neq y,|\alpha| \leq \mathrm{m}-1,1 \leq \mathrm{j} \leq \mathrm{D}\right\},
$$

where each $\xi_{\alpha, y, z, j} \in W^{m}(S)^{*}$ is characterized by the action

$$
\xi_{\alpha, y, z, j}\left[\left(\vec{P}^{x}\right)_{x \in S}\right]=\frac{\partial^{\alpha}\left(P_{j}^{y}-P_{j}^{z}\right)(y)}{|y-z|^{m-|\alpha|}} .
$$

Then the norm $\|\cdot\|_{\dot{W}^{m}(S)}$ can be computed via the formula

$$
\left\|\left(\overrightarrow{\mathrm{P}}^{x}\right)_{x \in S}\right\|_{\dot{W}^{m}(S)}=\sup _{\substack{\xi \in H(S) \\\|\xi\|_{W^{m}(S)^{*}} \leq 1}} \xi\left[\left(\vec{P}^{x}\right)_{x \in S}\right] .
$$

Mirroring (4.1), we define the selection "seminorm" to be

$$
\left\|\left(K_{x}\right)_{x \in S}\right\|_{\dot{W}^{m}(S)}:=\inf \left\{\left\|\left(\vec{P}^{x}\right)_{x \in S}\right\|_{\dot{W}^{m}(S)}: \vec{P}^{x} \in K(x) \text { for all } x \in S\right\} .
$$

We repeat proof of Theorem 1.4 with $\mathbb{X}=\mathrm{C}^{\mathrm{m}}\left(\mathbb{R}^{n}, \mathbb{R}^{\mathrm{D}}\right)$ in the previous section, but with the following modifications.

- We use $\|\cdot\|_{\mathbf{W}^{\mathfrak{m}(S)}}$ in place of $\|\cdot\|_{W^{m}(S)}$ (both the Whitney seminorm and the selection "seminorm").

- All the linear functionals will be chosen from $\mathrm{H}(S) \subset \mathrm{W}^{\mathrm{m}}(\mathrm{S})^{*}$.

- The map L in (4.4) will be replaced by a suitable linear map $\tilde{L}: H(S) \rightarrow \mathbb{R}^{\tilde{k}}$ for some $\tilde{k} \in \mathbb{N}$.

This concludes all the necessary modifications for the proof of Theorem 1.4 with $\mathbb{X}=\dot{\mathrm{C}}^{\mathrm{m}}\left(\mathbb{R}^{\mathrm{n}}, \mathbb{R}^{\mathrm{D}}\right)$.

The proof of Theorem 1.4 is complete. 


\section{Vector-Valued Shape Fields Finiteness Principle}

In this section we use what is colloquially known as the "gradient trick" to prove Theorem 2.4 using the $\mathrm{D}=1$ case proven in [19]. (See [19,20].)

The following proof will require working in both $\mathbb{R}^{n}$ and $\mathbb{R}^{n+D}$, so we provide a brief introduction to some of the notation.

The variable for $\mathbb{R}^{n}$ will be $x$, while $\mathbb{R}^{n+D}$ will be viewed as $\{z=(x, \xi): x \in$ $\left.\mathbb{R}^{n}, \xi \in \mathbb{R}^{D}\right\}$. The appropriate level of regularity for $\mathbb{R}^{n+D}$ will be $C^{m+1}$, so let $\mathcal{P}^{+}$denote the vector space of $\mathbb{R}$-valued, m-degree polynomials over $\mathbb{R}^{n+D}$. (Recall that $\overrightarrow{\mathcal{P}}$ is the vector space of $\mathbb{R}^{\mathrm{D}}$-valued, $(m-1)$-degree polynomials over $\mathbb{R}^{n}$.)

Proof of Theorem 2.4. Let $\mathrm{E}, \mathrm{Q}_{0} \subset \mathbb{R}^{\mathrm{n}},(\vec{\Gamma}(\mathrm{x}, \mathrm{M}))_{\mathrm{x} \in \mathrm{E}, \mathrm{M}>0}, \mathrm{C}_{w}, 0<\delta_{\mathrm{Q}_{0}} \leq \delta_{\max }, \mathrm{x}_{0} \in$ $E \cap 5 Q_{0}$ as in the hypotheses of Theorem 2.4 be given.

Let $\mathrm{E}^{+}=\{(x, 0): x \in E\} \subset \mathbb{R}^{n+D}$. For $\left(x_{0}, 0\right) \in E^{+}$, define

$$
\Gamma\left(\left(x_{0}, 0\right), M\right)=\left\{\mathrm{P} \in \mathcal{P}^{+}: \mathrm{P}(x, 0)=0, \nabla_{\xi} \mathrm{P}(x, 0) \in \vec{\Gamma}\left(x_{0}, M\right)\right\} .
$$

We now show that $(\Gamma(z, M))_{z \in E+}$ satisfies the hypotheses of the $D=1$ case of Theorem 2.4.

Let $S^{+} \subset E^{+}$with $\left|S^{+}\right| \leq k^{\sharp}$. By definition, $S^{+}$is of the form $\{(x, 0): x \in S\}$ for some $S \subset E$ with $|S| \leq k^{\sharp}$.

By hypothesis of Theorem 2.4 there exist $\left(\overrightarrow{\mathrm{P}}^{\mathrm{x}}\right)_{x \in S}$ such that

$$
\left\|\left(\overrightarrow{\mathrm{P}}^{\mathrm{x}}\right)_{x \in S}\right\|_{\dot{W}^{\mathrm{m}}(S)} \leq M_{0} \text {. }
$$

and

$$
\overrightarrow{\mathrm{P}}^{x} \in \vec{\Gamma}\left(x, M_{0}\right) \text { for all } x \in S .
$$

For $z=\left(x_{0}, 0\right) \in \mathrm{E}^{+}$, define

$$
P^{z}(x, \xi)=P^{\left(x_{0}, 0\right)}(x, \xi):=\sum_{j=1}^{D} \xi_{j} P_{j}(x) .
$$

Clearly, $\mathrm{P}^{\left(\mathrm{x}_{0}, 0\right)}\left(\mathrm{x}_{0}, 0\right)=0$ and $\nabla_{\xi} \mathrm{P}^{\left(\mathrm{x}_{0}, 0\right)}=\overrightarrow{\mathrm{P}}^{\mathrm{x}_{0}}$, so $\mathrm{P}^{z} \in \Gamma\left(z, \mathrm{M}_{0}\right)$ for all $z \in \mathrm{E}^{+}$. Let $\left(x_{0}, 0\right),\left(y_{0}, 0\right) \in E^{+}$. Then,

$$
\partial_{x}^{\alpha} P^{\left(x_{0}, 0\right)}(x, 0)=0 \text { and } \partial_{x}^{\alpha} \partial_{\xi}^{\beta} P^{\left(x_{0}, 0\right)}(x, 0)=0 \text { for }|\beta| \geq 2
$$

by definition, and for $1 \leq j \leq D$,

$$
\begin{aligned}
\left|\partial_{x}^{\alpha} \partial_{\xi_{j}}\left(P^{\left(x_{0}, 0\right)}-P^{\left(y_{0}, 0\right)}\right)\left(x_{0}, 0\right)\right| & =\left|\partial_{x}^{\alpha}\left(P_{j}^{x_{0}}-P_{j}^{y_{0}}\right)\left(x_{0}, 0\right)\right| \\
& \leq C\left|x_{0}-y_{0}\right|^{m-|\alpha|} \\
& =C\left|\left(x_{0}, 0\right)-\left(y_{0}, 0\right)\right|^{(m+1)-(|\alpha|+1)} .
\end{aligned}
$$

Thus, $\left(\mathrm{P}^{z}\right)_{z \in S}+$ satisfy $(2.3)$.

To demonstrate $\left(\mathrm{C}_{w}, \delta_{\max }\right)$-convexity, let $0<\delta \leq \delta_{\max }, x \in \mathrm{S}^{+}, \mathrm{M}<\infty, \mathrm{P}_{1}, \mathrm{P}_{2}, \mathrm{Q}_{1}, \mathrm{Q}_{2} \in$ $\mathcal{P}^{+}$be as in Definition 2.2. If $\mathrm{P}:=\mathrm{Q}_{1} \odot{ }_{\left(\mathrm{x}_{0}, 0\right)} \mathrm{Q}_{1} \odot_{\left(\mathrm{x}_{0}, 0\right)} \mathrm{P}_{1}+\mathrm{Q}_{2} \odot{ }_{\left(\mathrm{x}_{0}, 0\right)} \mathrm{Q}_{2} \odot{ }_{\left(\mathrm{x}_{0}, 0\right)} \mathrm{P}_{2}$, then $\mathrm{P}\left(\mathrm{x}_{0}, 0\right)=0$ and

$$
\nabla_{\xi} \mathrm{P}(\mathrm{x}, 0)=\left[\mathrm{Q}_{1} \odot_{\left(\mathrm{x}_{0}, 0\right)} \mathrm{Q}_{1} \odot_{\left(\mathrm{x}_{0}, 0\right)} \nabla_{\xi} \mathrm{P}_{1}\right](\mathrm{x}, 0)+\left[\mathrm{Q}_{2} \odot_{\left(\mathrm{x}_{0}, 0\right)} \mathrm{Q}_{2} \odot_{\left(\mathrm{x}_{0}, 0\right)} \nabla_{\xi} \mathrm{P}_{2}\right](\mathrm{x}, 0),
$$

which lies in $\Gamma\left(x, C_{w} M\right)$ by the $\left(C_{w}, \delta_{\max }\right)$-convexity of the $\vec{\Gamma}(x, M)$. 
Let $\mathrm{Q}^{\prime}$ be the unit cube in $\mathbb{R}^{\mathrm{D}}$. By the $\mathrm{D}=1$ case of Theorem 2.4 applied to $\mathrm{E}^{+} \subset \mathbb{R}^{n+\mathrm{D}},(\Gamma(z, M))_{z \in E+, M>0},\left(x_{0}, 0\right), \mathrm{Q}_{0} \times \mathrm{Q}^{\prime}$, we have the following. There exist $\mathrm{F} \in \mathrm{C}^{\mathrm{m}+1}\left(\mathbb{R}^{\mathrm{n}+\mathrm{D}}, \mathbb{R}\right)$ and $\mathrm{P}^{0} \in \Gamma\left(\left(\mathrm{x}_{0}, 0\right), \mathrm{CM}_{0}\right)$ such that

$$
\begin{gathered}
\mathrm{J}_{(x, 0)} \mathrm{F} \in \Gamma((x, 0), \mathrm{CM}) \text { for all }(x, 0) \in \mathrm{E}^{+} \\
\left|\partial_{x}^{\alpha} \partial_{\xi}^{\beta}\left(F-\mathrm{P}^{0}\right)(x, \xi)\right| \leq \mathrm{CM}_{0} \text { for all }(x, \xi) \in \mathrm{Q}_{0} \times \mathrm{Q}^{\prime},|\alpha|+|\beta| \leq \mathrm{m}+1 ;
\end{gathered}
$$

and

$$
\text { In particular, }\left|\partial_{x}^{\alpha} \partial_{\xi}^{\beta} F(x, \xi)\right| \leq \mathrm{CM}_{0} \text { for }|\alpha|+|\beta|=m+1 \text {. }
$$

Define $\overrightarrow{\mathrm{G}}(\mathrm{x}):=\nabla_{\xi} \mathrm{F}(\mathrm{x}, 0)$ and $\overrightarrow{\mathrm{Q}}^{0}(\mathrm{x})=\nabla_{\xi} \mathrm{P}^{\mathrm{0}}(\mathrm{x}, 0)$. We claim $\overrightarrow{\mathrm{G}} \in \mathrm{C}^{\mathrm{m}}\left(\mathbb{R}^{\mathrm{n}}, \mathbb{R}^{\mathrm{D}}\right)$ and $\vec{Q}^{0} \in \vec{\Gamma}(x, C M)$ are the desired function and jet, respectively, found in the conclusion of Theorem 2.4.

First, by (5.10),

$$
J_{x} G(y)=\nabla_{\xi} J_{(y, 0)} F(x, 0) \in \vec{\Gamma}(x, C M)
$$

because $J_{(y, 0)} F(x, 0) \in \vec{\Gamma}((x, 0), C M)$.

Next, for any $|\alpha| \leq m$ and $1 \leq j \leq D$,

$$
\begin{aligned}
\left|\partial_{x}^{\alpha}\left(G_{j}-Q_{j}^{0}\right)(x)\right| & =\left|\partial_{x}^{\alpha}\left(\partial_{\xi_{j}} F-\partial_{\xi_{j}} P^{0}\right)(x, 0)\right| \\
& \leq C M_{0} \delta_{Q_{0}}^{(m+1)-(|\alpha|+1)}=\mathrm{CM}_{0} \delta_{Q_{0}}^{m-|\alpha|}
\end{aligned}
$$

by (5.11).

Lastly, for $|\alpha|=m$,

$$
\left|\partial_{\chi}^{\alpha} G_{j}(x)\right|=\left|\partial_{\chi}^{\alpha} \partial_{\xi_{j}} F\right| \leq C M_{0}
$$

via (5.12).

\section{APPEndix A. Linear PRogramming AND DUALITY}

Lemma A.1 (LP Duality Theorem). Let $\mathrm{p}, \mathrm{q}$ be positive integers. Let $\mathrm{c} \in \mathbb{R}^{\mathrm{p}}$ and $\mathrm{b} \in \mathbb{R}^{\mathrm{q}}$. Let $\mathrm{A}: \mathbb{R}^{\mathrm{p}} \rightarrow \mathbb{R}^{\mathrm{q}}$ be a linear map. Consider the following two optimization problems.

$$
\begin{gathered}
\text { Maximize } \mathrm{c}^{\top} \cdot \mathrm{x} \text { subject to } \mathrm{A} \mathrm{x} \leq \mathrm{b} \\
\text { Minimize } \mathrm{b}^{\top} \cdot \mathrm{y} \text { subject to } \mathrm{A}^{\top} \mathrm{y}=\mathrm{c} \text { and } \mathrm{y} \geq 0
\end{gathered}
$$

Suppose one of (A.1) or (A.2) has a feasible solution, then both have feasible and optimal solutions. Moreover, if $x_{0}$ optimizes (A.1) and $y_{0}$ optimizes (A.2), then $\mathrm{c}^{\top} \cdot \mathrm{x}_{0}=\mathrm{b}^{\top} \cdot \mathrm{y}_{0}$, i.e. the maximum of A.1 equals the minimum of (A.2).

The same conclusion holds if we replace " $\mathrm{Ax} \leq \mathrm{b}$ " by " $\mathrm{Ax} \leq \mathrm{b}$ and $\mathrm{x} \geq 0$ " in (A.1) and " $\mathrm{A}^{\top} \mathrm{y}=\mathrm{c}$ " by " $\mathrm{A}^{\top} \mathrm{y} \geq \mathrm{c}$ " in (A.2).

See 24] for a proof.

We generalize the theorem above to finite dimensional normed spaces.

Lemma A.2. Let $\mathrm{V}$ be a finite-dimensional normed vector space with norm $\|\cdot\|_{\mathrm{V}}$ and dual $\mathrm{V}^{*}$. Let $\mathrm{L}: \mathrm{V}^{*} \rightarrow \mathbb{R}^{\mathrm{q}}$ be a linear map and let $\mathrm{L}^{*}: \mathbb{R}^{\mathrm{q}} \rightarrow \mathrm{V}$ be the dual operator of $\mathrm{L}$ defined by $1 \mathrm{l}$

$$
\chi^{\top} \cdot \mathrm{L}(\phi)=\left\langle\phi, \mathrm{L}^{*} x\right\rangle \text { for all } \chi \in \mathbb{R}^{\mathrm{q}} \text { and } \phi \in \mathrm{V}^{*} .
$$

\footnotetext{
${ }^{1}$ Here we identify the dual of any Euclidean space with itself via the dot product.
} 
Let $\mathrm{b} \in \mathbb{R}^{\mathrm{q}}$. Suppose there exists $\phi_{0} \in \mathrm{V}^{*}$ such that $\mathrm{L}\left(\phi_{0}\right) \leq \mathrm{b}$. Then

$$
\sup _{\mathrm{L}(\phi) \leq \mathrm{b}}\langle\phi, v\rangle=\inf _{\substack{y \geq 0 \\ L^{*} y=v}} b^{\top} \cdot y .
$$

Proof. Let $p=\operatorname{dim} V<\infty$. There exists a linear isomorphism $J: V \rightarrow \mathbb{R}^{p}$. Let $J^{*}: \mathbb{R}^{p} \rightarrow \mathrm{V}^{*}$ denote its dual. Note that $\mathrm{J}^{*}$ is also a linear isomorphism. We have the following diagram.

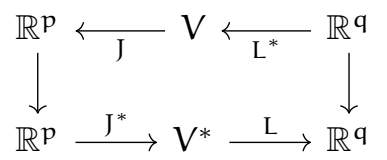

For each $v \in \mathrm{V}$ and $\phi \in \mathrm{V}^{*}$, there exist unique $\mathrm{c}, x \in \mathbb{R}^{\mathrm{p}}$ such that $\mathrm{J}^{-1}(\mathrm{p})=v$ and $\mathrm{J}^{*}(\mathrm{x})=\phi$. Thus, thanks to LP Duality Theorem (see Appendix), we have

$$
\begin{aligned}
\sup _{\mathrm{L}(\phi) \leq \mathrm{b}}\langle\phi, v\rangle & =\sup _{\operatorname{LoJ}(\mathrm{x}) \leq \mathrm{b}}\left\langle J^{*}(\mathrm{x}), J^{-1}(\mathrm{c})\right\rangle \\
& =\sup _{\operatorname{LoJ^{*}}(\mathrm{x}) \leq \mathrm{b}} \mathrm{c}^{\top} \cdot \mathrm{x} \\
& =\inf _{\substack{\left(\operatorname{LoJ}^{*}\right)^{\top} y=c \\
y \geq 0}} \mathrm{~b}^{\top} \cdot \mathrm{y} .
\end{aligned}
$$

Notice that $\left(L \circ J^{*}\right)^{\top}=J \circ L^{*}$. Moreover, since $J$ is an isomorphism, the equality $\mathrm{J} \circ \mathrm{L}^{*} \mathrm{y}=\mathrm{c}$ is equivalent to $\mathrm{L}^{*} \mathrm{y}=\mathrm{J}^{-1}(\mathrm{c})=v$. A.3. follows.

\section{REFERENCES}

[1] Edward Bierstone and Pierre D. Milman. $\mathcal{e}^{\mathrm{m}}$-norms on finite sets and $\mathrm{e}^{\mathrm{m}}$-extension criteria. Duke Mathematical Journal, 137(1):1-18, 2007.

[2] Edward Bierstone, Pierre D. Milman, and Wiesław Pawłucki. Differentiable functions defined in closed sets. A problem of Whitney. Invent. Math., 151(2):329-352, 2003.

[3] Edward Bierstone, Pierre D. Milman, and Wiesław Pawłucki. Higher-order tangents and fefferman's paper on Whitney's extension problem. Ann. of Math. (2), 164(1):361-370, 2006.

[4] Yuri Brudnyi and Pavel Shvartsman. A linear extension operator for a space of smooth functions defined on a closed subset in $\mathbb{R}^{n}$. Dokl. Akad. Nauk SSSR, 280(2):268-272, 1985.

[5] Yuri Brudnyi and Pavel Shvartsman. Generalizations of Whitney's extension theorem. Internat. Math. Res. Notices, 3(129), 1994.

[6] Yuri Brudnyi and Pavel Shvartsman. The traces of differentiable functions to subsets of $\mathbb{R}^{n}$. In Linear and complex analysis. Problem book 3, volume 1574 of Lecture Notes in Mathematics, pages 279-281. Springer-Verlag, Berlin, 1994.

[7] Yuri Brudnyi and Pavel Shvartsman. The Whitney problem of existence of a linear extension operator. J. Geom. Anal., 7(4):515-574, 1997.

[8] Yuri Brudnyi and Pavel Shvartsman. The trace of jet space $\mathrm{J}^{\mathrm{k}} \wedge^{\omega}$ to an arbitrary closed subset of $\mathbb{R}^{\mathrm{n}}$. Trans. Amer. Math. Soc., 350(4):1519-1553, 1998.

[9] Yuri Brudnyi and Pavel Shvartsman. Whitney's extension problem for multivariate $C^{1, \omega_{-}}$ functions. Trans. Amer. Math. Soc., 353(6):2487-2512 (electronic), 2001.

[10] Charles Fefferman. A generalized sharp Whitney theorem for jets. Rev. Mat. Iberoam., 21(2):577-688, 2005.

[11] Charles Fefferman. Interpolation and extrapolation of smooth functions by linear operators. Rev. Mat. Iberoam., 21(1):313-348, 2005.

[12] Charles Fefferman. A sharp form of Whitney's extension theorem. Ann. of Math. (2), 161(1):509-577, 2005.

[13] Charles Fefferman. Whitney's extension problem for $\mathrm{C}^{\mathrm{m}}$. Ann. of Math. (2), 164(1):313-359, 2006.

[14] Charles Fefferman. $C^{m}$ extension by linear operators. Ann. of Math. (2), 166(2):779-835, 2007. 
[15] Charles Fefferman. The structure of linear extension operators for $\mathrm{C}^{\mathrm{m}}$. Rev. Mat. Iberoam., 23(1):269-280, 2007.

[16] Charles Fefferman. Fitting a $\mathrm{C}^{\mathrm{m}}$-smooth function to data III. Ann. of Math. (2), 170(1):427441, 2009.

[17] Charles Fefferman. Whitney's extension problems and interpolation of data. Bull. Amer. Math. Soc. (N.S.), 46(2):207-220, 2009.

[18] Charles Fefferman. The $\mathrm{C}^{\mathrm{m}}$ norm of a function with prescribed jets. Rev. Mat. Iberoam., 26(3):1075-1098, 2010.

[19] Charles Fefferman, Arie Israel, and Garving K. Luli. Finiteness principles for smooth selections. Geom. Funct. Anal., 26(2):422-477, 2016.

[20] Charles Fefferman and Garving K. Luli. The Brenner-Hochster-Kollár and Whitney problems for vector-valued functions and jets. Rev. Mat. Iberoam., 30(3):875-892, 2014.

[21] Charles Fefferman and Pavel Shvartsman. Sharp finiteness principles for lipschitz selections. Geom. Funct. Anal., 28:1641-1705, 2018.

[22] Georges Glaeser. Étude de quelques algèbres tayloriennes. J. Analyse Math., 6:1-124, 1958.

[23] Elias M. Stein. Singular Integrals and Differentiability Properties of Functions, volume 2 of Monographs in harmonic analysis. Princeton University Press, Princeton, NJ, 1970.

[24] J. Matousek and B. Gärtner. Understanding and Using Linear Programming. Universitext. Springer Berlin Heidelberg, 2007.

[25] Pavel Shvartsman. Traces of functions of zygmund class. Sibirsk. Mat. Zh., 5:203-215, 1987.

[26] Pavel Shvartsman. Lipschitz selections of set-valued mappings and Helly's theorem. J. Geom. Anal., 12(2):289-324, 2002.

[27] Pavel Shvartsman. The Whitney extension problem and Lipschitz selections of set-valued mappings in jet-spaces. Trans. Amer. Math. Soc., 360(10):5529-5550, 2008.

[28] Pavel Shvartsman. On Lipschitz selections of affine-set valued mappings. Geom. Funct. Anal., 11(4):2001, 840-868.

[29] Hassler Whitney. Analytic extensions of differentiable functions defined in closed sets. Trans. Amer. Math. Soc., 36(1):63-89, 1934.

[30] Hassler Whitney. Differentiable functions defined in closed sets. I. Trans. Amer. Math. Soc., 36(2):369-387, 1934.

[31] Hassler Whitney. Functions differentiable on the boundaries of regions. Ann. of Math. (2), 35(3):482-485, 1934.

Department of Mathematics, UC Davis One Shields Ave Davis, CA 95616

Email address: fsjiang@math.ucdavis.edu

Department of Mathematics, UC Davis One Shields Ave Davis, CA 95616

Email address: kluli@math.ucdavis.edu

Department of Mathematics, UC Davis One Shields Ave Davis, CA 95616

Email address: oneill@math.ucdavis.edu 